\title{
Knowledge, Attitude, Practice, and Fear of COVID-19: an Online-Based Cross-cultural Study
}

\author{
Mohammad Ali ${ }^{1,2}$ - Zakir Uddin ${ }^{3,4}$ - Palash Chandra Banik ${ }^{5}$ (D) \\ Fatma A. Hegazy ${ }^{6}$ (D) . Shamita Zaman ${ }^{7}$. Abu Saleh Mohammed Ambia ${ }^{8}$. \\ Md. Kaoser Bin Siddique ${ }^{9}$ (D) . Rezoana Islam ${ }^{10}$. Fatema Khanam ${ }^{11}$. \\ Sayed Mohammad Bahalul ${ }^{12} \cdot$ Md Ahiduzzaman Sharker $^{13} \cdot$ FMAkram Hossain $^{14}$. \\ Gias U. Ahsan ${ }^{15}$ (D)
}

Accepted: 23 August 2021 / Published online: 30 August 2021

(C) The Author(s), under exclusive licence to Springer Science+Business Media, LLC, part of Springer Nature 2021

\begin{abstract}
The study aimed to measure Knowledge, Attitude, and Practice (KAP) and fear level towards COVID-19 and explore its cross-cultural variances in knowledge by sociodemographic factors among the general population of 8 different countries over 5 continents. It was a cross-sectional online survey. This survey was conducted in April 2020 among 1296 participants using the "Google Form" platform. Considering the social distancing formula and pandemic situation, we collect data using popular social media networks. Univariate and bivariate analyses were used to explore the collected data on KAP, fear, and sociodemographic factors. Overall knowledge score was $9.7 \pm 1.7$ (out of 12), and gender differences (female vs male: $9.8 \pm 1.6$ vs $9.5 \pm 1.9)$ were significant $(p=0.008)$ in the bivariate analysis. Knowledge score variances were found significant in some regions by gender, marital status, and education qualification. The highest and lowest mean knowledge scores were recorded in the Middle East $(10.0 \pm 1.7)$ and Europe $(9.3 \pm 2.0)$. Despite having a high fear score $(22.5 \pm 5.6$ out of 35$), 78.35 \%$ of respondents were positively and $81.7 \%$ in a good practice level. Fear score rankings: Middle East (1st; $23.8 \pm 5.5)$, Europe (2nd; $23.2 \pm 5.8$ ), Africa (3rd; $22.7 \pm 5.0$ ), South Asia (4th; $22.1 \pm 5.7)$, Oceania (5th; 21.9 \pm 5.8 ), and North America (6th; 21.7 \pm 5.5 ). Fear and knowledge were not correlated. KAP and fear variation exist among geographical regions. Gender, marital status, and education qualification are factors in knowledge variances for some regions. KAP and fear measures can help health education programs consider some sociodemographic factors and regions during an outbreak of highly contagious disease and uplift a positive attitude and good practice.
\end{abstract}

Keywords COVID-19 $\cdot$ Knowledge $\cdot$ Attitude $\cdot$ Practice $\cdot$ Fear $\cdot$ Cross-cultural study Online survey

Mohammad Ali

alibup2018@gmail.com

Extended author information available on the last page of the article 
COVID-19 disease caused by a novel coronavirus (SARS-CoV-2) has been declared as a pandemic by the World Health Organization (WHO) on March 11, 2020 (Bedford et al., 2020). Towards the end of April, it has been reported that the virus has spread across many countries around the world with fear-evoking death reports. (Johns Hopkins Coronavirus Resource Center, 2020). This virus is highly contagious with some symptoms such as fever, dry cough, fatigue, myalgia, and dyspnea (Zhong et al., 2020). However, the human carrier of the virus without any clinical symptoms can also spread to other humans (Cascella, Rajnik, Cuomo, Dulebohn, \& Di Napoli, 2020). The mortality rate due to this virus is higher than common contagious diseases and can reach up to 15\% (Baud et al., 2020). Due to these unique features, an adequate management strategy is needed to battle the virus and save lives.

Effective pandemic management requires support from the population at risk and necessary measures to alleviate disease spread. Previous studies of a similar kind of contagious disease, for example, the H1N1 flu outbreak, have revealed that Knowledge, Attitudes, and Practices (KAP) play a significant role in personal experience and effect behaviors during pandemic (Yap et al., 2010). Other studies have shown that improved knowledge has increased the uptake of preventive measures and influences the protective behavior at the individual and community level (Lau et al., 2007; Leung et al., 2005).

KAP is the major adherence factor for successfully implementing prevention and control measures for COVID-19 (Ajilore et al., 2017; Tachfouti et al., 2012). Knowledge and attitudes have greater impacts on emotions, personality, anxiety, panic, and stress (Hinthorn, 1997). These attributes also influence the preventive measures against infectious diseases and healthcare services' utilization due to fear and stigma (Person et al., 2004; Siddique et al., 2017). Studies on previous epidemics indicated that some people experience "fear" during a serious disease outbreak, requiring support from public health professionals (Hinthorn, 1997). Quarantine and isolation are necessary to reduce the spread of the virus. There is evidence of increased fear during quarantine and isolation at the global and country-level (Jefferson et al., 2008). In fact, "fear" is a psychophysiological construct linking to consciousness's neurobiology (Adolphs, 2013). A person may fail to think rationally and clearly with the cognitive load when reacting to a pandemic with a higher fear level. An individual's fear of COVID-19 level may help healthcare providers design an appropriate program for better management.

Despite having a unique character of the COVID-19, the number of identified infected cases varied from country to country. Nonetheless, most of the countries around the world announced lockdown as an emergency measure to ensure people stay at home and maintain social distancing. Despite the same preventive measures taken by the governments of high-income countries, several countries in Europe and the USA have been seriously hit by the COVID-19 pandemic. However, low- and middle-income countries such as Bangladesh and Egypt reported fewer identified cases (Johns Hopkins Coronavirus Resource Center, 2020). Therefore, it would be interesting to consider whether KAP and fear levels are different between high-income and middle-income countries. There is a research gap combining KAP and fear towards COVID-19. The novelty of this study is exploring cross-cultural KAP and fear issues by collecting data from 8 different countries over 5 continents around the globe during the peak global pandemic period in April. This study aimed to (1) measure KAP and fear level towards COVID-19 and explore its cross-cultural variances among the general population of Australia, Bangladesh, Canada, Egypt, Sweden, UAE, UK, and the USA and (2) examine the effect of cross-cultural knowledge level towards COVID-19 on sociodemographic factors (e.g., age, gender, marital status, educational qualification, and occupational status). 


\section{Methods and Materials}

\section{Study Settings and Participants}

An online cross-sectional survey was conducted from April 6 to 27, 2020, using a "Google Form" platform. Social media platforms such as Facebook, Facebook Messenger, WhatsApp, and Skype were used for data collection because of the COVID-19 pandemic situation. These platforms are popular worldwide and a convenient platform to reach many people within a short time. The survey invitation was sent to social media contacts with the Authors living in different countries. This Google Form was an open invitation to social media users, and the posts were repeated every three days as a reminder to the participants. We could not calculate the response rate as it was simultaneously shared by the authors and their social media friends (those who are the existing Facebook and WhatsApp friends of the investigators). Our participants include both male and female population aged 16 years and above and willingly took part in the survey. Current or recovered COVID-19 patients and healthcare workers/volunteers working in management or control programs were excluded. Respondents from other than eight chosen countries were also excluded. The survey form contained a brief introduction to the background, objective, and procedures. The survey was voluntary, anonymous, and confidential. As it was a multi-continental study, we used the English language for this survey; however, we requested the respondents used Google Translate if required. We got the response from 1296 invitees, and after considering inclusion and exclusion criteria, we include 1255 participants for data analysis.

All the continent and subcontinent were selected purposively in the study, but the countries were chosen for data collection using lottery methods to ensure the study's representativeness to avoid the confounding effect of socioeconomic and cultural issues. Primarily, we targeted two countries from each continent. However, except for Europe and North America, we received data from one country due to the investigators' lack of links. Overall, we received 1296 respondents from all the continents and subcontinents within predefined data collection days (total 21 days). However, we have to discard 41 data for their incompleteness. Finally, we got 1255 data for the analysis and the distributions of the data were as follow: South Asia, Bangladesh $(n=545)$; Oceania, Australia $(n=111)$; North America, Canada $(n=69)$ and USA $(n=54)$; Europe, Sweden $(n=49)$ and UK $(n=72)$; Middle East, UAE $(n=225)$; and Africa, Egypt $(n=130)$.

Ethical Approval The ethical approval was taken from the Ethics Committee of North South University, Bangladesh (the institutional review board registration number: NSUIRB-20-013,023). Informed consent was taken using the "Google Form" platform before data collection. According to the Helsinki declaration, all the concerns related to biomedical research were followed strictly throughout the study, and consent was assumed as completing the survey questions. Participants were informed that their participation was voluntary and that they could withdraw from the study at any point or choose not to answer any question. Participants' confidentiality was maintained as no identifying information was collected, and findings were disseminated only in aggregate. 


\section{Measurements}

The online survey questionnaire had three parts. The first part consisted of sociodemographic variables, including age, gender, marital status, education, occupation, and country of current residence.

In the second part, we used a questionnaire to assess the knowledge, attitude, and practice toward COVID-19 developed by B. L. Zhong et al., (2020). This questionnaire had 16 individual questions; among them, the first 12 questions represented the knowledge part. These 12 questions were answered on a true/false basis with an additional option of "I don't know." A correct answer was given 1 point, and an incorrect/unknown answer was given 0 points. The total knowledge score ranged from 0 to 12 , with a higher score explaining a better knowledge of COVID-19. The middle two questions were used to measure attitude toward COVID-19, which was scored the same as knowledge questions. The last two questions with yes/no answers were used to assess the respondents' practice toward COVID-19. The level of knowledge was measured using the Bell curved approach (mean $\pm 1 \mathrm{SD}$ ). The level of knowledge was classified as "Poor" (less than Mean - 1SD), "Average" (Mean-SD to Mean + 1SD), and "Good" (Mean +1SD).

In the third part, fear was measured by the Fear of Coronavirus-19 Scale (FCV-19S) developed by Ahorsu et al. (2020). Seven items were included in the FCV-19S (e.g., "I am most afraid of coronavirus-19") measuring the fear of the respondents on COVID-19. Participants were asked to rate their agreement with the statement using a 5-point Likert scale and the following options, and the scores were 1= "Strongly Disagree," 2 = "Disagree," $3=$ "Neither Agree and/or Nor Disagree," 4= "Agree," and "5 = Strongly Agree." The minimum score for each question was 1 , and the maximum was 5. A total score was calculated by adding up each item score (ranging from 7 to 35). The higher the score considered the greater the fear of coronavirus-19. The scale has a robust psychometric property. It is a reliable $(\alpha=0.82$ and ICC $=0.72)$ and valid scale for the general population in assessing fear of COVID-19 (Ahorsu et al., 2020).

\section{Statistical Analysis}

First of all, data was managed and cleaned as well as logically checked for internal consistency. Some of the data were discarded for their incompleteness or their inconsistency. The statistical analysis part was divided into two fundamental parts. The first part was the univariate analysis. The frequency, percentage, mean, and standard deviation (SD) were done as appropriate to describe the knowledge, attitudes, practice, and fear scores according to the respondents' sociodemographic characteristics. The second part was the bivariate analysis, where the independent sample's $t$-test, one-way analysis of variance (one-way ANOVA), was done as appropriate to see the mean difference within the groups. Multiple linear regression analysis was performed and whereas level of knowledge was considered as dependent variables and sociodemographic variables considered as independent variables. Data analyses were conducted with SPSS software version 25.0. The statistical significance level was set at $p<0.05$ (two-tailed). For data analysis, we assigned the respondents according to their greater continental/subcontinental geographical region: South Asia (Bangladesh), Oceania (Australia), North America (Canada and USA), Europe (Sweden and UK), Middle East (UAE), and Africa (Egypt). To explore the cross-cultural issue, all the data is presented into the tables and graphs by the respondents' area of residence. 


\section{Results}

\section{Trajectories of "Knowledge" Score by Cross-cultural Sociodemographic Factors}

Table 1 shows the sociodemographic characteristics in comparison to the knowledge score. Overall, male and female ratios were 1:1.1, and more than half of the respondents were in the age group of 16 to 29 years $(52.6 \%)$ and married $(51.2 \%)$. The majority of $(84.6 \%)$ of the respondents' educational qualification was graduation and above. Almost three out of five respondents were employed (58.8\%) in any way. The total mean knowledge score was 9.6 (out of 12), whereas the highest and lowest mean knowledge score was recorded in the Middle East (10.0) and Europe (9.3), respectively.

We found some variances in knowledge by sociodemographic factors. The overall mean knowledge score by (1) age was 9.6, whereas the highest and lowest mean knowledge score were recorded in Middle East (10.2) and South Asia (9.2), respectively, but the difference was not significant within the age group of the inter region; (2) gender was 9.7, whereas the highest and lowest mean knowledge scores were recorded in the Middle East (9.9) and European (9.3) regions, respectively, and the male and female knowledge score difference was significant for overall $(p=0.008)$ and Oceania ( $p=0.007)$ region; (3) marital status was 9.6, whereas the highest and lowest mean knowledge scores were recorded in Middle East (10.0) and Europe (9.1), respectively, and the knowledge score difference among the married, never married, and others was significant for North American $(p=0.007)$; (4) educational qualification was 9.3, and it was significant $(p<0.001)$, whereas the highest and lowest mean knowledge score was recorded in Middle East (9.7) and Europe (8.9), respectively, and the knowledge score difference between different level of education was significant for South Asia $(p<0.001)$, Oceania $(p=0.025)$, Meddle East $(p=0.009)$, and African $(p=0.036)$ regions; (5) occupational status was 9.6, whereas the highest and lowest mean knowledge score was recorded in Europe (9.9) and Middle East (9.0), respectively, and the difference was not significant for their different types of jobs.

\section{Percentage of Correct Responses and Level of "Knowledge" by Area of Residence}

Figure 1a shows the distribution of correct responses by geographical area. The mean $( \pm \mathrm{SD})$ of the total score was $9.7( \pm 1.7)$ out of 12 possible knowledge scores. We classified knowledge scores as poor (less than Mean -SD), average (Mean - SD to Mean +SD), and good (more than Mean $+\mathrm{SD}$ ). Therefore, for "poor knowledge," the cut-off was less than 8.0; for "average knowledge," the cut-off was 8.0-11.4, and for the "good knowledge," the cut-off was more than 11.4. The highest percentage of respondents with good knowledge was reported in Europe (18.2\%) region, whereas lowest was reported in Oceania $(2.7 \%)$ and the difference was $15.5 \%$. Besides, the highest percentage of respondents with average knowledge was reported in Oceania (86.5\%) region, whereas lowest was reported in Africa $(75.7 \%)$ and the difference was $10.8 \%$; as well as, the highest percentage of respondents with poor knowledge was reported in Africa (14.2\%) region, whereas lowest was reported in Europe $(5.0 \%)$ and the difference was $9.2 \%$. Percentage of correct responses of Knowledge by area of residence can be found in supplementary file (Table 3). Regarding the factors associated with poor COVID 19 knowledge, marital status (overall, North America and South Asia region), occupation (Africa and North America region), and gender (North 


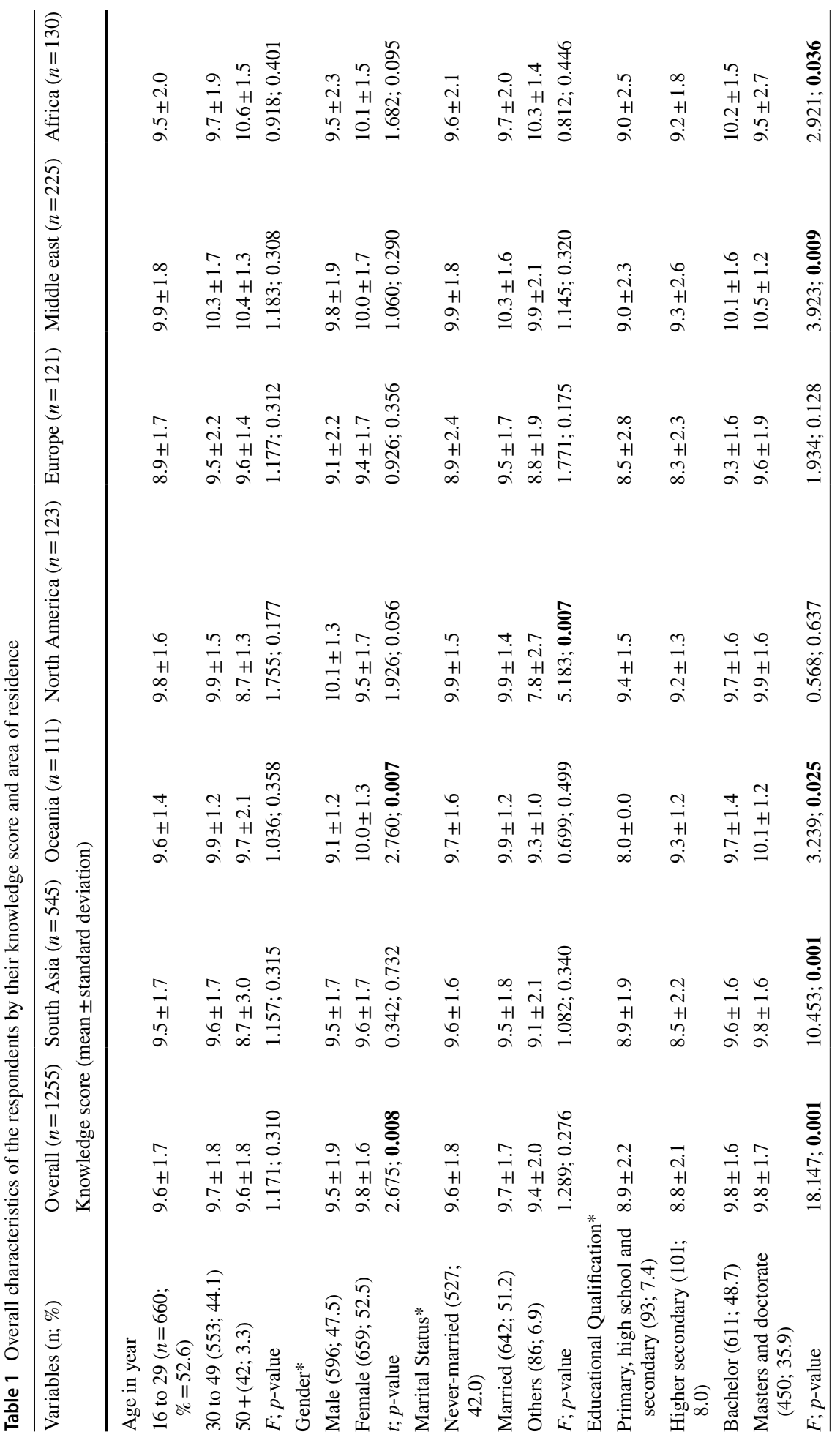




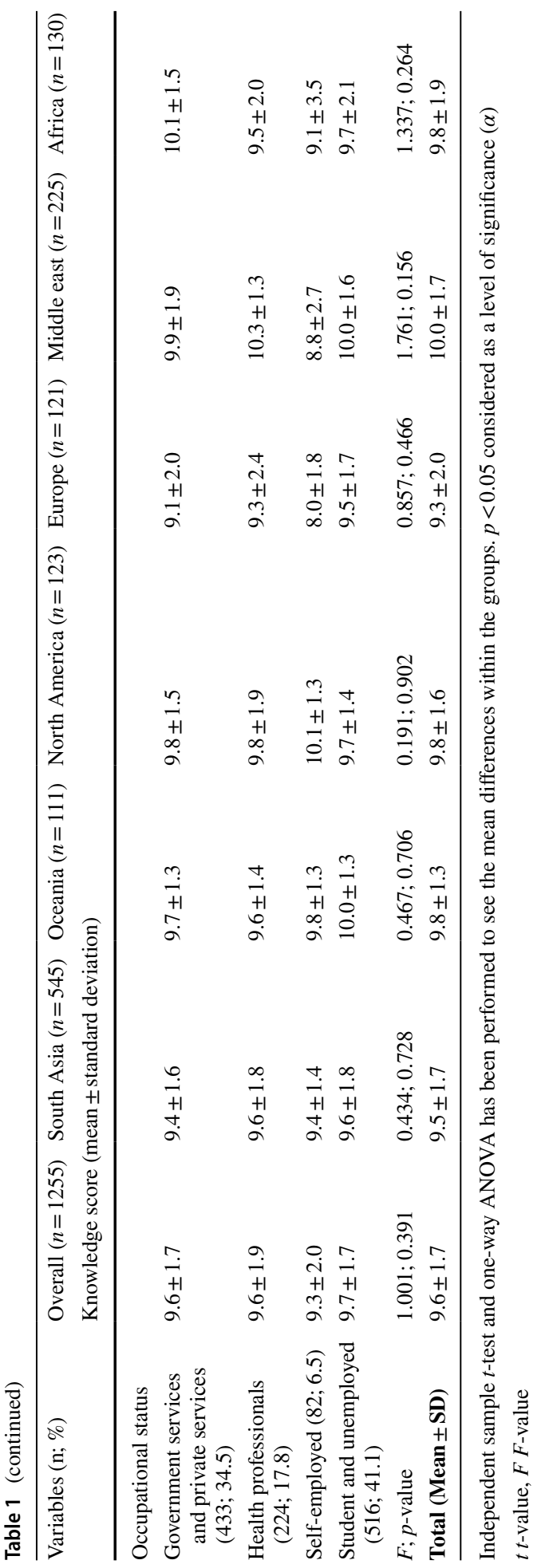




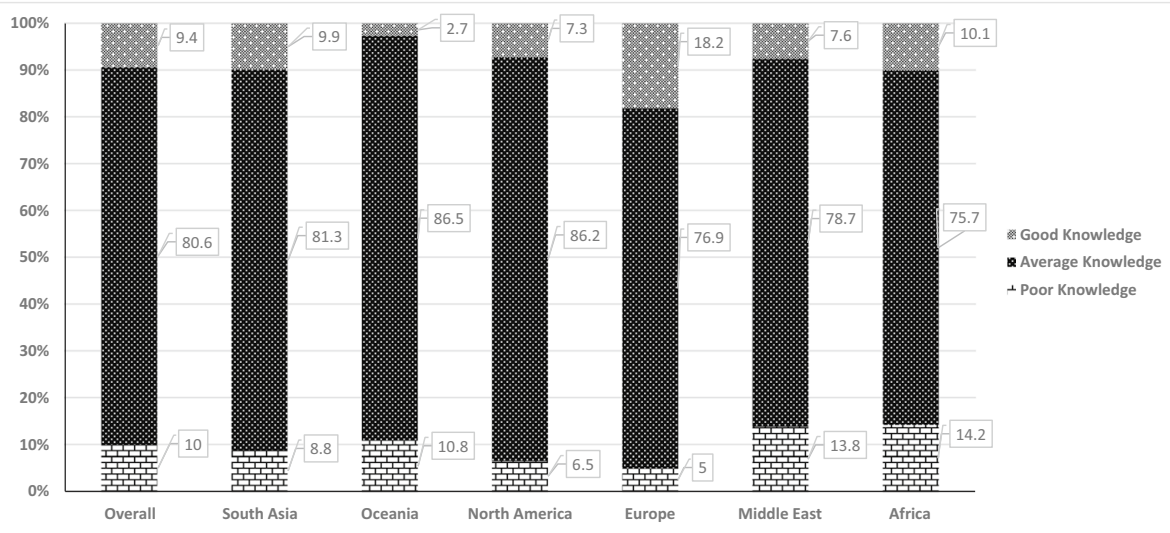

(a) Level of knowledge according to classified knowledge score by geographical area ( $n=1255$ )

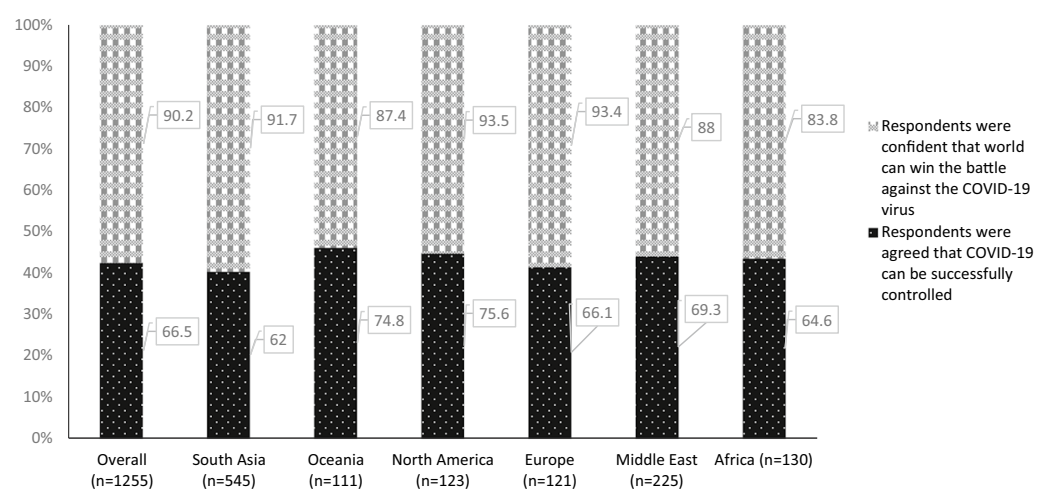

(b) Attitudes of the respondents towards COVID 19 according to the area of residence.

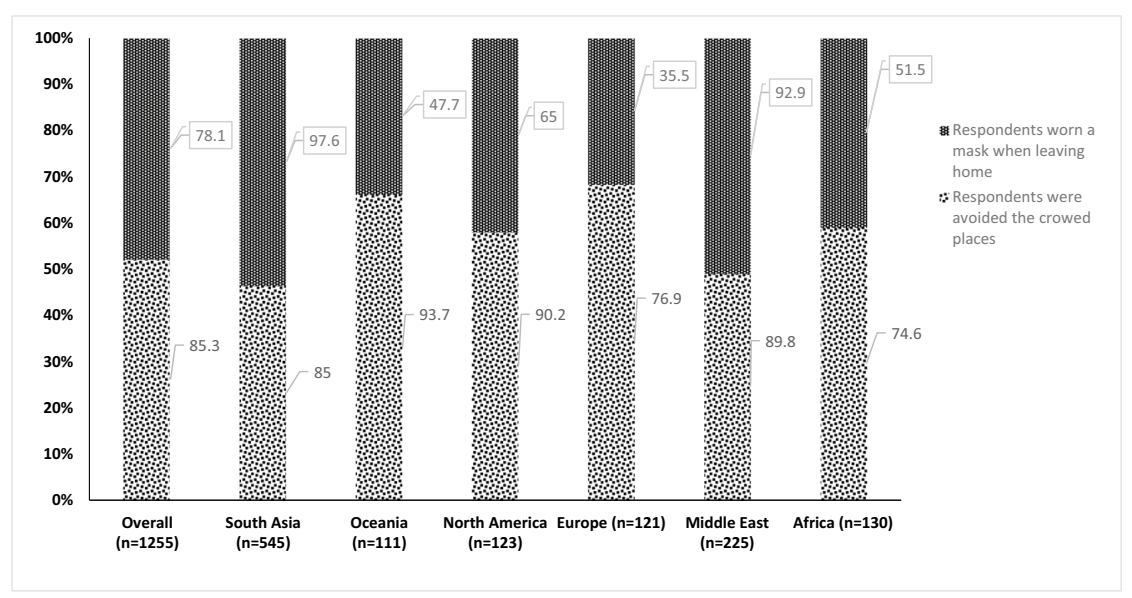

(c) Practices of the respondents towards COVID 19 according to the area of residence.

Fig. 1 a Level of knowledge according to classified knowledge score by geographical area $(n=1255)$. b Attitudes of the respondents towards COVID 19 according to the area of residence. c Practices of the respondents towards COVID 19 according to the area of residence 
America region) have been reported as associated factors but these factors were not significant as shown in multiple linear regression (Supplementary file Table 4).

\section{Percentage of Correct Responses of "Attitudes" and "Practices" by Area of Residence}

The highest correct response for the 1st question regarding attitudes was recorded in North America (93.5\%), and the lowest correct response was recorded in Africa (83.8\%) region, and the difference was $9.7 \%$. The highest correct response for the 2 nd question regarding attitudes was recorded in North America (75.6\%), and the lowest correct response was recorded in South Asia (62.0\%) region, and the difference was $13.6 \%$ (Fig. 1b).

The highest correct response was recorded in South Asia (97.6\%), and the lowest correct response was recorded in Oceania $(47.7 \%)$ region, and the difference was significant (49.9\%). The second question was whether the respondents avoided crowded places? And the highest correct response was recorded in Oceania $(93.7 \%)$, and the lowest correct response was recorded in the Africa (74.6\%) region, and the difference was $19.1 \%$ (Fig. 1c).

\section{"Fear" by Geographical Region}

Regarding the fear related to COVID-19, three out of ten (30.5\%) respondents responded positively about fear. Respondents from South Asia reported the highest positive responses in fear $(34.8 \%)$, whereas respondents from the Middle East reported the lowest positive responses in fear $(22.7 \%)$, and the response difference was $12.1 \%$. When we assessed their mean score for the fear, we found that the highest mean score for fear was in the Middle East (Ranked, 1st; 23.8 \pm 5.5 ), which indicates greater fear of coronavirus-19 compared to other's continents like Europe (Ranked, 2nd; 23.2 \pm 5.8 ), Africa (Ranked, 3rd; 22.7 \pm 5.0 ), South Asia (Ranked, 4th; 22.1 \pm 5.7 ), Oceania (Ranked, 5th; 21.9 \pm 5.8 ), and North America (Ranked, 6th; 21.7 \pm 5.5 ), but the difference was minimum (Table 2).

\section{Discussion}

In our study, a mixed knowledge score was observed for different demographic variables within the different regions. However, the knowledge score mean was relatively better in the Middle East and relatively poor in the European region. The marital status, occupation, and gender factors were associated with knowledge in the bivariate analysis. South Asian respondents had the highest positive responses in fear, but they ranked 4th. Respondents from the Middle East reported the lowest positive responses in fear, but they ranked 1st by the Fear level. This indicates that the respondents from the Middle East had a greater fear of COVID-19. We did not find a correlation between knowledge and fear scores. This signifies that fear is not dependent on the respondent's knowledge of COVID-19.

In this study, most of the participants were young adults and middle-aged, and more than half of the respondents were female. Interestingly, the knowledge score towards COVID-19 was higher among the female, and the overall score was statistically significant. In the Oceania region, females' level of knowledge is better than males, followed by Africa, the Middle East, Europe, and South Asia. However, in North America, the level of knowledge is higher among males than in females. Like our study, Chinese females scored higher in the knowledge section when a KAP study towards COVID-19 was conducted in 


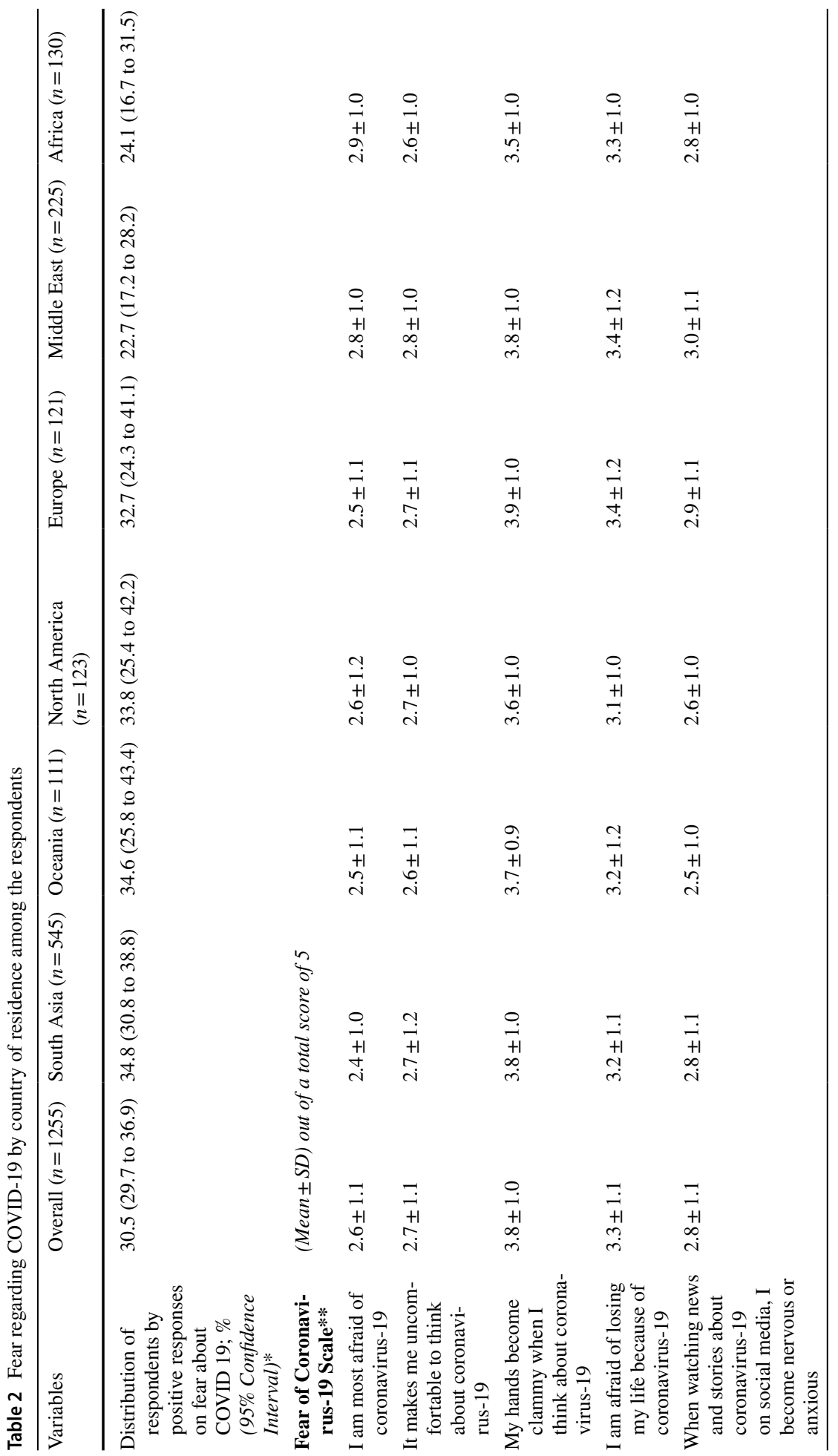

\section{Springer}




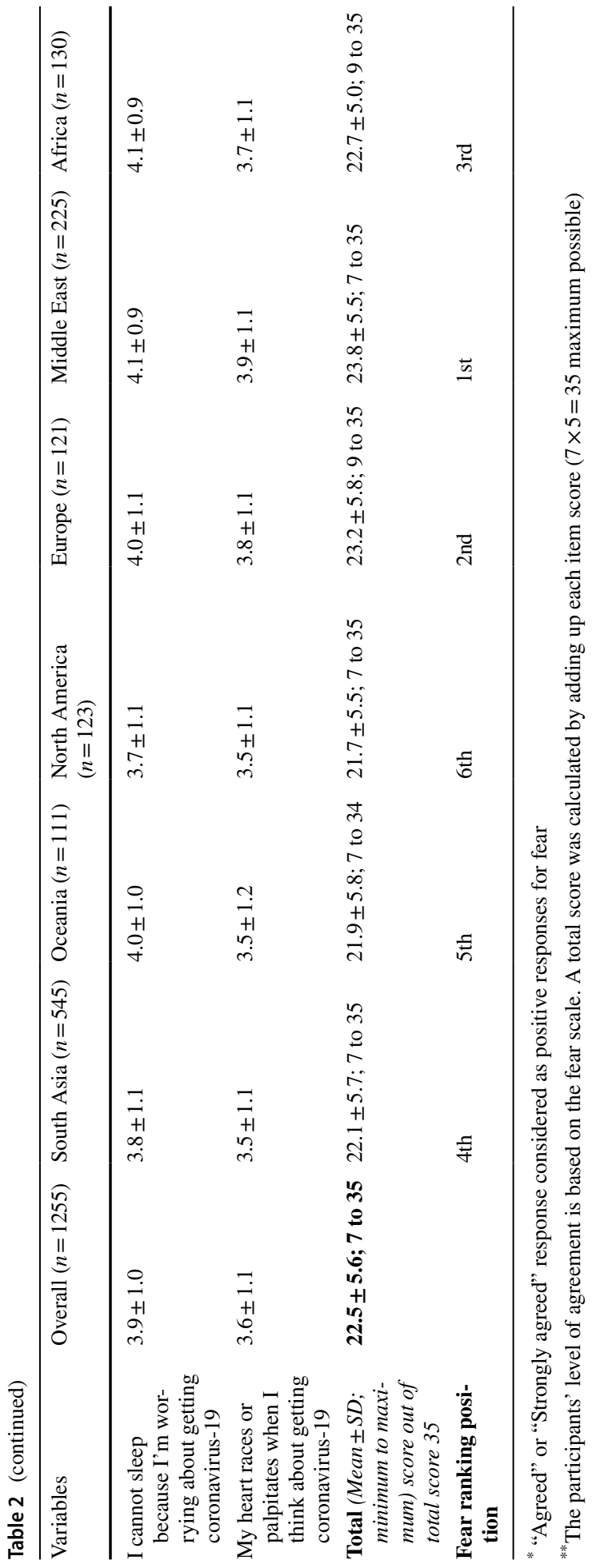


China (Zhong et al., 2020). Interestingly, according to recent statistics, there is a higher prevalence of male patients diagnosed with COVID-19 than females (Jin et al., 2020; Vahidy et al., 2021).

The educated population dominated our study. Predictably, knowledge regarding COVID-19 was higher in the graduate and postgraduate participants. Especially in the South Asian and Oceania region, participants who did attend university are scored lower. These findings were consistent with other studies where the authors found poor knowledge about the infectious disease among non-graduate populations (Nwagbara et al., 2021; Wang et al., 2018). We found significant differences in overall and Middle East regional knowledge scores by educational status.

Previous studies found that the spread rate of infectious diseases is higher among those who did not have good knowledge about the disease (Goni et al., 2019; Rassi et al., 2019). In our study, we found the lowest knowledge score among participants of the highly affected European region. The comparative picture of the rate of COVID-19 infection during our survey period (Johns Hopkins Coronavirus Resource Center, 2020) and knowledge level distribution in our result (e.g., good, medium, and poor) is somehow mixed. The reason might be the nature of the previous infectious disease conditions, and this global pandemic condition is quite different. Participant's regional social norm or cultural issue on mass media exposure might be different for getting information on this pandemic.

In China, most of the respondents held an optimistic attitude towards the COVID-19 epidemic: $90.8 \%$ believed that COVID-19 would be successfully controlled, and $97.1 \%$ had confidence that China can win the battle against the virus (Zhong et al., 2020). Nonetheless, when we measure the attitude towards this pandemic, $90.2 \%$ of the respondents were confident that the world would win the battle against COVID-19; however, overall, only $60 \%$ of participants were optimistic that this disease could be successfully controlled. The region-wise upbeat attitude was almost the same.

Despite a severe attack by COVID-19 in Europe, we found only $35.5 \%$ of Europeans wore a mask when going outside. This rate was significantly lower than the overall practice rate $(78.1 \%)$ of wearing masks when they went out. The wearing mask rate was also considerably lower among participants living in another severely affected North American region. Our study result supports the reality of limiting contagious disease through good practice behaviors (Buckee et al., 2021; Squibb \& Yardley, 1999).

Previous scientific study has shown that fear of disease negatively affected public health efforts to control the disease outbreak. (Person et al., 2004). Our study found a higher fear score among the Middle East participants and followed by Europe and Africa. The highest positive responses in fear found in South Asia. Region-specific cultural issues and mass media exposure frequency (e.g., news on death due to COVID 19) might be a reason for this fear. However, we did not find a correlation between the respondents' knowledge and fear scores in our study.

Limitations of the online survey cannot be ruled out in our study. Due to limited access to the internet, old age and rural people who have been more likely to have poor KAP were not included in the study. Selection bias may be significant if those of poor level KAP people appear to have less participation. KAP for vulnerable populations deserves special research attention. Moreover, there is a risk of bias due to online data collection. Ethical challenge is a fact in the cross-cultural study (Durham, 2014). However, we have mitigated the challenge for this study by maintaining some measures, such as (1) collecting informed consent before participating in the survey, (2) depersonalizing responders, and (3) ensuring confidentiality for better personal security measures like other surveys in public health (Survey to Identify the Public Health Ethics Needs of Public Health Practitioners in 
Canada: Preliminary Results, 2018). This study's findings could not be generalized as we cannot ensure the representative sample size and sampling technique. Our study used overall mean and SD for the knowledge classification, which may potentially confound effect.

Despite having those limitations, cross-cultural nature is the main strength of this study. It is difficult to compare this study to other similar kinds of studies. To our knowledge, no study measured the cross-cultural value of KAP and fear of COVID-19. Therefore, the result of this study is a novel contribution to the scientific literature. This study imposes a chance to evaluate the relationship between KAP and infection numbers among the population in a different continent. A representative from several professions and all ages made the study more generalized.

\section{Conclusion}

The majority of the study participants show an "average" level of knowledge (overall $80.6 \%$ according to this study's knowledge level classification). Future cross-cultural study is needed to clarify the fact and find a way to improve a "good" level of knowledge. It will help guide better educational health campaign strategies targeting global people by considering cultural/regional aspects. This study suggests that mass media health education programs aimed at improving knowledge and reducing fear could encourage an optimistic attitude and maintain safe practices that might result in a controlled infection rate by creating awareness among the global population considering their culture or regional social norm. KAP and fear measures can help; health education programs consider some sociodemographic factors and geographical regions during an outbreak of highly contagious disease.

Supplementary Information The online version contains supplementary material available at https://doi. org/10.1007/s11469-021-00638-4.

Acknowledgements All the authors acknowledge the participants for providing us with the information to conduct the study. The authors also would like to thank Hasan Hafizur Rahman from Canada, Ifty Mahmud from Australia, and Md. Ahsan Ullah Shah Masud from Bangladesh for helping in data collection.

Author Contribution Mohammad Ali: Conceptualization, Methodology, Investigation, Data curation, Data analysis conceptualization, Writing — original draft, Writing_review and editing, Validation. Zakir Uddin: Conceptualization, Investigation, Formal data analysis, Writing-review and editing, Supervision, Validation. Palash Chandra Banik: Data curation, Formal data analysis, Writing-result interpretation draft, Writing—review and editing, Validation. Fatma A. Hegazy: Writing—review, Validation. Shamita Zaman: Writing-English language usage, grammar, and spelling, review and editing, Validation. Abu Saleh Mohammed Ambia: Writing-English language usage, grammar, and spelling, review and editing, Validation. Md. Kaoser Bin Siddique: Writing_introduction draft, Writing_review, Validation. Rezoana Islam: Writing — review, Validation. Fatema Khanam: Writing — review, Validation. Sayed Mohammad Bahalul: Writing—review, Validation. Md Ahiduzzaman Sharker: Writing—review, Validation. F M Akram Hossain: Writing review, Validation. Gias U Ahsan: Supervision, Validation.

Data Availability The data sets used and analyzed for this study are available. Please feel free to ask the corresponding author. We have also provided some supplementary data.

\section{Declarations}

Ethical Approval We conducted the study according to the guidelines laid down in the Declaration of Helsinki, and all procedures involving human subjects were approved by the Institutional Review Board (IRB) of Uttara Adhunik Medical College (UAMC-IRB-20-013023). Informed consent was taken using the "Google 
Form" platform before participating in the online survey. The survey was voluntary, anonymous, and confidential.

Consent for Publication Not applicable.

Competing Interests The authors declare no competing interests.

\section{References}

Adolphs, R. (2013). The biology of fear. Current Biology, 23(2), R79-R93. https://doi.org/10.1016/j. cub.2012.11.055

Ahorsu, D. K., Lin, C.-Y., Imani, V., Saffari, M., Griffiths, M. D., \& Pakpour, A. H. (2020). The fear of COVID-19 scale: Development and initial validation. International Journal of Mental Health and Addiction. https://doi.org/10.1007/s11469-020-00270-8

Ajilore, K., Atakiti, I., \& Onyenankeya, K. (2017). College students' knowledge, attitudes and adherence to public service announcements on Ebola in Nigeria: Suggestions for improving future Ebola prevention education programmes. Health Education Journal, 76(6), 648-660. https://doi.org/10. $1177 / 0017896917710969$

Baud, D., Qi, X., Nielsen-Saines, K., Musso, D., Pomar, L., \& Favre, G. (2020). Real estimates of mortality following COVID-19 infection. The Lancet Infectious Diseases. https://doi.org/10.1016/ S1473-3099(20)30195-X

Bedford, J., Enria, D., Giesecke, J., Heymann, D. L., Ihekweazu, C., Kobinger, G., ... Wieler, L. H. (2020, March 28). COVID-19: Towards controlling of a pandemic. The Lancet, 395, 1015-1018. https://doi.org/10.1016/S0140-6736(20)30673-5

Buckee, C., Noor, A., \& Sattenspiel, L. (2021). Thinking clearly about social aspects of infectious disease transmission. Nature 2021 595:7866, 595(7866), 205-213. https://doi.org/10.1038/ s41586-021-03694-X

Cascella, M., Rajnik, M., Cuomo, A., Dulebohn, S. C., \& Di Napoli, R. (2020). Features, evaluation and treatment coronavirus (COVID-19). In StatPearls. Retrieved from http://www.ncbi.nlm.nih.gov/ pubmed/32150360

Durham, J. (2014). Ethical challenges in cross-cultural research: A student researcher's perspective. Australian and New Zealand Journal of Public Health, 38(6), 509-512. https://doi.org/10.1111/17536405.12286

Goni, H., Naing, W.-A., Deris, A., \& Baaba. . (2019). Assessment of knowledge, attitude and practice towards prevention of respiratory tract infections among Hajj and Umrah Pilgrims from Malaysia in 2018. International Journal of Environmental Research and Public Health, 16(22), 4569. https:// doi.org/10.3390/ijerph16224569

Hinthorn, D. R. (1997). Quarantine! East European Jewish Immigrants and the New York City Epidemics of 1892. Annals of Internal Medicine, 127(10), 950. https://doi.org/10.7326/0003-4819-127-10199711150-00022

Jefferson, T., Foxlee, R., Del Mar, C., Dooley, L., Ferroni, E., Hewak, B., ... Rivetti, A. (2008). Cochrane Review: Interventions for the interruption or reduction of the spread of respiratory viruses. Evidence-Based Child Health: A Cochrane Review Journal, 3(4), 951-1013. https://doi.org/10.1002/ ebch. 291

Jin, J.-M., Bai, P., He, W., Wu, F., Liu, X.-F., Han, D.-M., .. Yang, J.-K. (2020). Gender differences in patients with COVID-19: Focus on severity and mortality. Frontiers in Public Health, 8, 152. https:// doi.org/10.3389/fpubh.2020.00152

Johns Hopkins Coronavirus Resource Center. (2020). Retrieved from https://coronavirus.jhu.edu/

Lau, J. T. F., Kim, J. H., Tsui, H., \& Griffiths, S. (2007). Anticipated and current preventive behaviors in response to an anticipated human-to-human H5N1 epidemic in the Hong Kong Chinese general population. BMC Infectious Diseases, 7(1), 18. https://doi.org/10.1186/1471-2334-7-18

Leung, G. M., Ho, L.-M., Chan, S. K. K., Ho, S.-Y., Bacon-Shone, J., Choy, R. Y. L., ... Fielding, R. (2005). Longitudinal assessment of community psychobehavioral responses during and after the 2003 outbreak of severe acute respiratory syndrome in Hong Kong. Clinical Infectious Diseases, 40(12), 1713-1720. https://doi.org/10.1086/429923 
Nwagbara, U. I., Osual, E. C., Chireshe, R., Bolarinwa, O. A., Saeed, B. Q., Khuzwayo, N., \& Hlongwana, K. W. (2021). Knowledge, attitude, perception, and preventative practices towards COVID-19 in subSaharan Africa: A scoping review. PLoS ONE, 16(4), e0249853. https://doi.org/10.1371/JOURNAL. PONE.0249853

Person, B., Sy, F., Holton, K., Govert, B., Liang, A., Garza, B., ... Zauderer, L. (2004). Fear and stigma: The epidemic within the SARS Outbreak. Emerging Infectious Diseases, 10(2), 358-363. https://doi. org/10.3201/eid1002.030750

Rassi, C., Martin, S., Graham, K., de Cola, M. A., Christiansen-Jucht, C., Smith, L. E., ... Massangaie, M. (2019). Knowledge, attitudes and practices with regard to schistosomiasis prevention and control: Two cross-sectional household surveys before and after a Community Dialogue intervention in Nampula province, Mozambique. PLOS Neglected Tropical Diseases, 13(2), e0007138. https://doi.org/10.1371/ journal.pntd.0007138

Siddique, M. K. Bin, Islam, S. M. S., Banik, P. C., \& Rawal, L. B. (2017). Diabetes knowledge and utilization of healthcare services among patients with type 2 diabetes mellitus in Dhaka, Bangladesh. BMC Health Services Research, 17(1). https://doi.org/10.1186/s12913-017-2542-3

Squibb, B., \& Yardley, K. (1999). Playing healthy, staying healthy: A prevention program for contagious disease. Early Childhood Education Journal, 26, 143-147. https://doi.org/10.1023/A:1022973216955

Survey to identify the public health ethics needs of public health practitioners in Canada: Preliminary results. (n.d.). Retrieved from http://www.ncchpp.ca/127/Publications.ccnpps?id

Tachfouti, N., Slama, K., Berraho, M., \& Nejjari, C. (2012). The impact of knowledge and attitudes on adherence to tuberculosis treatment: A case-control study in a moroccan region. Pan African Medical Journal, 12(1). https://doi.org/10.11604/pamj.2012.12.52.1374

Vahidy, F. S., Pan, A. P., Ahnstedt, H., Munshi, Y., Choi, H. A., Tiruneh, Y., .. McCullough, L. D. (2021). Sex differences in susceptibility, severity, and outcomes of coronavirus disease 2019: Cross-sectional analysis from a diverse US metropolitan area. PLOS ONE, 16(1), e0245556. https://doi.org/10.1371/ JOURNAL.PONE.0245556

Wang, M., Han, X., Fang, H., Xu, C., Lin, X., Xia, S., ... Tao, H. (2018). Impact of health education on knowledge and behaviors toward infectious diseases among students in Gansu Province, China. BioMed Research International, 2018, 1-12. https://doi.org/10.1155/2018/6397340

Yap, J., Lee, V. J., Yau, T. Y., Ng, T. P., \& Tor, P.-C. (2010). Knowledge, attitudes and practices towards pandemic influenza among cases, close contacts, and healthcare workers in tropical Singapore: A cross-sectional survey. BMC Public Health, 10(1), 442. https://doi.org/10.1186/1471-2458-10-442

Zhong, B.-L., Luo, W., Li, H.-M., Zhang, Q.-Q., Liu, X.-G., Li, W.-T., \& Li, Y. (2020a). Knowledge, attitudes, and practices towards COVID-19 among Chinese residents during the rapid rise period of the COVID-19 outbreak: A quick online cross-sectional survey. International Journal of Biological Sciences, 16(10), 1745-1752. https://doi.org/10.7150/ijbs.45221

Publisher's Note Springer Nature remains neutral with regard to jurisdictional claims in published maps and institutional affiliations.

\title{
Authors and Affiliations
}

\author{
Mohammad Ali $^{1,2}$ (Dakir Uddin ${ }^{3,4}$ (D) Palash Chandra Banik ${ }^{5}$ (D) \\ Fatma A. Hegazy ${ }^{6}$ (D) . Shamita Zaman ${ }^{7}$. Abu Saleh Mohammed Ambia ${ }^{8}$. \\ Md. Kaoser Bin Siddique ${ }^{9}$ (D) . Rezoana Islam ${ }^{10} \cdot$ Fatema Khanam $^{11}$. \\ Sayed Mohammad Bahalul ${ }^{12} \cdot$ Md Ahiduzzaman Sharker $^{13} \cdot$ FMAkram Hossain $^{14}$. \\ Gias U. Ahsan ${ }^{15}$ (D)
}

Zakir Uddin

uddinz2@mcmaster.ca

Palash Chandra Banik

palashcbanik@gmail.com

Fatma A. Hegazy

fhegazy@sharjah.ac.ae 
Shamita Zaman

shamita.zaman48@gmail.com

Abu Saleh Mohammed Ambia abu.ambia2@rbwm.gov.uk

Md. Kaoser Bin Siddique

kaoserbd@gmail.com

Rezoana Islam

rezoanashibly@gmail.com

Fatema Khanam

fatemakhanam33@yahoo.com

Sayed Mohammad Bahalul smbahalul@gmail.com

Md Ahiduzzaman Sharker dr.ahiduzzaman@gmail.com

FMAkram Hossain

fm_akram@yahoo.com

Gias U. Ahsan

gias.ahsan@northsouth.edu

1 Department of Physiotherapy and Rehabilitation, Uttara Adhunik Medical College and Hospital, Uttara, Dhaka, Bangladesh

2 Centre for Higher Studies and Research, Bangladesh University of Professionals, Dhaka, Bangladesh

3 School of Rehabilitation Sciences, McMaster University, Hamilton, ON, Canada

4 School of Physical and Occupational Therapy, McGill University, Montreal, QC, Canada

5 Department of Noncommunicable Diseases, Bangladesh University of Health Sciences, Dhaka, Bangladesh

6 Department of Physiotherapy, College of Health Sciences, University of Sharjah, Sharjah, UAE

7 Department of Public Health, Macquarie University, Macquarie Park, New South Wales, Australia

8 Short Term Support and Rehabilitation Services, Optalis, Royal Borough of Windsor \& Maidenhead, Maidenhead, UK

9 Essential Healthcare for the Disadvantaged in Bangladesh Project, Disabled Rehabilitation and Research Association, Dhaka, Bangladesh

10 Department of Physical Therapy, University of Toronto, Toronto, Canada

11 Karoliniska Institute, Stockholm, Sweden

12 Department of Physiotherapy, Sundsvall Hospital, Sundsvall, Sweden

13 Division of Public Health, Manitoba Health, MB Government, Manitoba, Canada

14 Department of Physical Therapy, Citi medical services, New York, NY, USA

15 Department of Public Health, North South University, Basundhara, Dhaka, Bangladesh 